\title{
Dimensional versus cut-off renormalization and the nucleon-nucleon interaction
}

\author{
Angsula Ghosh, ${ }^{1}$ Sadhan K. Adhikari, ${ }^{1}$ and B. Talukdar ${ }^{1,2}$ \\ ${ }^{1}$ Instituto de Física Teórica, Universidade Estadual Paulista, 01405-900 São Paulo, São Paulo, Brazil \\ ${ }^{2}$ Department of Physics, Visva Bharati, Santiniketan 731235, India \\ (Received 14 January 1998; revised manuscript received 13 July 1998)
}

\begin{abstract}
The role of dimensional regularization is discussed and compared with that of cut-off regularization in some quantum mechanical problems with ultraviolet divergence in two and three dimensions with special emphasis on the nucleon-nucleon interaction. Both types of renormalizations are performed for attractive divergent oneand two-term separable potentials, a divergent tensor potential, and the sum of a delta function and its derivatives. We allow energy-dependent couplings, and determine the form that these couplings should take if equivalence between the two regularization schemes is to be enforced. We also perform renormalization of an attractive separable potential superposed on an analytic divergent potential. [S0556-2813(98)07310-5]
\end{abstract}

PACS number(s): 21.30.-x, 03.65.Nk, 11.10.Gh, 13.75.Cs

\section{INTRODUCTION}

Ultraviolet divergences appear in exact as well as perturbative treatments of the nonrelativistic quantum mechanical two-body problem in momentum space interacting via twobody potentials with certain singular behavior at short distances [1-13] in two and three space dimensions. Similar divergences appear in perturbative quantum field theory and are usually treated by renormalization techniques $[14,15]$. There are several variants of renormalization that employ different types of regularizations, such as the cut-off, and dimensional regularizations. Unless there is some symmetry violation in performing regularization, in perturbative field theory, both regularization schemes are expected to lead to the same renormalized result at low energies. The closely related technique of discretization on the lattice in such field theoretic problems also should lead to equivalent results. Exactly as in quantum field theory, the ultraviolet divergences in quantum mechanics can be treated by renormalization. Three schemes have been used for the purpose: cut-off regularization [1-3,5-7,10,11], dimensional regularization $[6,9,11]$, and discretization on the lattice [4]. For the simplest $\delta$-function potential all three approaches lead to the identical result.

Recently, cut-off $[1,5-7,10,11]$ and dimensional $[6,10,11]$ regularizations have been successfully used by several workers in quantum mechanical problems in $S$ and higher partial waves in the context of nucleon-nucleon interaction. Although both regularization schemes have been successfully used for the purpose of renormalization in quantum mechanical problems with ultraviolet divergence, their equivalence cannot be demonstrated except in the simplest problems. In this work we consider several problems with ultraviolet divergence, allow energy-dependent bare couplings, and find the forms that these couplings must take in order to obtain equivalence between the two regularization schemes.

We consider four potentials for illustration. The simplest is the minimal potential in a general partial wave considered recently [1]. In momentum space this potential possesses only the threshold behavior and is given by the following one-term separable form:

$$
V(p, q)=p^{L} \lambda_{L} q^{L},
$$

with $L$ the angular momentum. For $L=0$, this potential is the usual $\delta$-function potential. As the Lippmann-Schwinger equation has the same generic form in all partial waves, the ultraviolet divergence of this potential becomes stronger and stronger as $L$ increases. Next we consider a two-term separable potential of the form

$$
V(p, q)=\lambda_{0} u(p) u(q)+\lambda_{2} v(p) v(q),
$$

where $u(p)$ and $v(p)$ are each divergent form factors of the type $p^{L}$, considered in the first potential. We also consider the potential $[5,6,10,11]$

$$
V(p, q)=\lambda_{1}+\lambda_{2}\left(p^{2}+q^{2}\right) .
$$

Potential (3) is the sum of a $\delta$ function and its second derivatives. This potential is interesting as it appears as a piece in the low-energy nucleon-nucleon potential derived from effective field theory and has received attention recently $[11,13]$. Finally, we consider the following minimal tensor nucleon-nucleon potential possessing ultraviolet divergence:

$$
\left|V_{L L^{\prime}}(p, q)\right| \equiv\left(\begin{array}{cc}
V_{00}(p, q) & V_{02}(p, q) \\
V_{20}(p, q) & V_{22}(p, q)
\end{array}\right)=\left(\begin{array}{cc}
\lambda_{0} & \lambda_{1} q^{2} \\
\lambda_{1} p^{2} & \lambda_{2} p^{2} q^{2}
\end{array}\right),
$$

where $\lambda_{0}$ and $\lambda_{2} p^{2} q^{2}$ are divergent $S$ and $D$ wave parts of the nucleon-nucleon potential (1). In Eq. (4) the angular momentum labels $L$ and $L^{\prime}$ are explicitly shown. The term involving $\lambda_{1}$ provides the $S$ - $D$ coupling. When $\lambda_{1}=0$, the $S$ and $D$ waves decouple and we essentially have potential (1). The functions $p^{2}$ and $q^{2}$ are the threshold factors for $L=2$.

The nucleon-nucleon potential derived from a chiral Lagrangian formulation of effective field theory contains usual finite-range potentials superposed on divergent potentials containing delta function and derivatives (gradients) and can be written as $[5,6,10,11]$

$$
\begin{aligned}
V(p, q)= & V_{f}(p, q)+\lambda_{1}+\lambda_{2}\left(p^{2}+q^{2}\right)+\lambda_{3} p^{2} q^{2} \\
& +\lambda_{4}\left(p^{4}+q^{4}\right)+\cdots,
\end{aligned}
$$


where $V_{f}(p, q)$ represents the usual finite-range parts of the potential. The configuration-space derivatives of the $\delta$ function appear as powers of momenta in momentum space. Potential (3) is just a part of Eq. (5). Though one can renormalize the divergent parts separately, it is not clear that a potential, such as Eq. (5), containing a divergent and a finiterange part can be successfully renormalized. Though a general answer to this question involving local finite-range potentials may involve a complicated numerical calculational scheme, we would like to address this point in a much simpler context, where we take the finite-range part to be an attractive separable potential frequently used to simulate the nucleon-nucleon interaction. We perform an analysis to show that when a divergent potential of the form $\lambda$ or $\lambda p^{2} q^{2}$ is summed to an attractive separable potential, the renormalized scattering $K$ or $t$ matrix leads to physically plausible results for the nucleon-nucleon system at low energies.

The plan of our work is as follows. We perform renormalization of potentials (1), (2), (3), and (4), in Secs. II A, II B, II C, and II D, respectively, using both dimensional and cutoff regularizations. In Sec. II A we consider the scattering problem in both two and three dimensions. Potentials (2), (3), and (4) are mostly of interest in nuclear physics and hence we shall be limited to only the three-dimensional case in Secs. II B, II C, and II D. In Sec. III we present results for the renormalization of a divergent potential of the form $\lambda$ or $\lambda p^{2} q^{2}$ added to an attractive separable potential. Finally, in Sec. IV a brief summary of the present work is presented.

\section{REGULARIZATION AND RENORMALIZATION}

The partial-wave Lippmann-Schwinger equation for the $K$ matrix $K_{L}\left(p, q, k^{2}\right)$, at center-of-mass energy $k^{2}$, is given, in dimension $d$, by

$$
\begin{aligned}
K_{L}\left(p, k, k^{2}\right)= & V_{L}(p, k)+\mathcal{P} \int q^{d-1} d q V_{L}(p, q) \\
& \times G\left(q ; k^{2}\right) K_{L}\left(q, k, k^{2}\right),
\end{aligned}
$$

with the free Green's function $G\left(q ; k^{2}\right)=\left(k^{2}-q^{2}\right)^{-1}$, in units $\hbar=2 m=1$, where $m$ is the reduced mass; $\mathcal{P}$ in Eq. (6) denotes principal value prescription for the integral and the momentum-space integration limits are from 0 to $\infty$. The (on-shell) scattering amplitude $t_{L}(k)$ is defined by

$$
\frac{1}{t_{L}(k)}=\frac{1}{K_{L}\left(k^{2}\right)}+i \frac{\pi}{2} k^{d-2},
$$

where $K_{L}\left(k^{2}\right) \equiv K_{L}\left(k, k, k^{2}\right)=-(2 / \pi)\left(\tan \delta_{L} / k\right)$ with $\delta_{L}$ the phase shift. All scattering observables can be calculated using $t_{L}(k)$. Though we are considering a general dimension $d$, we shall be limited in the present work to $d=2$ and 3 . Most of our results can be generalized to higher dimensions. The condition of unitarity is given by

$$
\operatorname{Im} t_{L}(k)=-\frac{\pi}{2} k^{d-2}\left|t_{L}(k)\right|^{2}
$$

where Im denotes the imaginary part. Here we employ a $K$-matrix description of scattering. Then the renormalization algebra will involve only real quantities and we do not have to worry about unitarity, which can be imposed later via Eq. (7). This is the simplest procedure to follow, as all renormalization schemes preserve unitarity.

\section{A. The minimal potential}

Minimal potential (1) has been renormalized by cut-off regularization in three dimensions in [1] using the $t$-matrix approach. Here we present a brief account of that work with appropriate generalization to the $K$-matrix approach in both two and three dimensions. We also perform dimensional regularization with this potential and discuss its consistency with cut-off regularization. For minimal potential (1), the $K$ matrix of Eq. (6) permits the following analytic solution:

$$
K_{L}\left(p^{\prime}, p, k^{2}\right)=p^{\prime L} \tau_{L}(k) p^{L},
$$

with the $\tau$ function defined by

$$
\begin{gathered}
\tau_{L}(k)=\left[\lambda_{L}^{-1}-I_{L}(k)\right]^{-1} \\
I_{L}(k)=\mathcal{P} \int q^{d-1} d q q^{2 L} G\left(q ; k^{2}\right) .
\end{gathered}
$$

Integral $I_{L}(k)$ of Eq. (11) possesses ultraviolet divergence. As the $\tau$ function completely determines the $K$ matrix and as the divergent terms are contained in it, we consider the renormalization of this function.

Because of the ultraviolet divergence in Eq. (11), some regularization is needed to give meaning to it. We use the following regularized Green's function with a sharp cutoff:

$$
G_{R}\left(q, \Lambda ; k^{2}\right)=\left(k^{2}-q^{2}\right)^{-1} \Theta(\Lambda-q),
$$

with $\Theta(x)=0$ for $x<0$ and $=1$ for $x>0$. In Eq. (12), $\Lambda$ $(\gg k)$ is a large but finite quantity. In the end, the limit $\Lambda$ $\rightarrow \infty$ has to be taken. Finite results for physical magnitudes, as $\Lambda \rightarrow \infty$, are obtained only if the coupling $\lambda_{L}$ is understood to be the so-called bare coupling $\lambda_{L}(k, \Lambda)$.

In order to proceed with the choice of the bare coupling we have to consider a specific value of dimension $d$. First, we consider $d=3$. The choice of the bare coupling can be found by inspection of the following regularized form of Eq. (11):

$$
\begin{aligned}
I_{R L}(k, \Lambda) & \equiv \mathcal{P} \int q^{2} d q q^{2 L} G_{R}\left(q, \Lambda ; k^{2}\right) \\
& =-\left[\sum_{j=0}^{L} \frac{k^{2(L-j)} \Lambda^{2 j+1}}{2 j+1}+\frac{k^{2 L+1}}{2} \ln \left|\frac{\Lambda-k}{\Lambda+k}\right|\right] .
\end{aligned}
$$

In the large $\Lambda(\gg k)$ limit, the logarithmic term in Eq. (14) tends to zero and

$$
\lim _{\Lambda \rightarrow \infty} I_{R L}(k, \Lambda)=-\sum_{j=0}^{L} \frac{k^{2(L-j)} \Lambda^{2 j+1}}{2 j+1} .
$$

All terms in Eq. (15) diverge as $\Lambda \rightarrow \infty$. Except for $L=0$, these divergent terms are momentum $(k)$ dependent. 
For obtaining a finite renormalized $\tau$ function, the coupling $\lambda_{L}$ should be understood to be the so-called bare coupling $\lambda_{L}(k, \Lambda)$. In Ref. [1] the following energy-dependent bare coupling was used:

$$
\lambda_{L}^{-1}(k, \Lambda)=-\sum_{i=0}^{L} \frac{k^{2(L-i)} \Lambda^{2 i+1}}{2 i+1}+\Lambda_{0 L}\left(k^{2}\right),
$$

where function $\Lambda_{0 L}\left(k^{2}\right)$ can be used to introduce the physical scale(s) of the system and characterizes the strength of the interaction. The quantity $1 / \Lambda_{0 L}\left(k^{2}\right)$ could be termed the renormalized strength or coupling of the interaction. We are taking the bare coupling to be energy dependent. In the present nonrelativistic context this is not of concern, as the bare coupling is not an observable. We shall see that the renormalized $K$ matrix obtained after renormalization from this bare coupling has the desired analytic properties in $k^{2}$. We shall see that this energy dependence is necessary, in order to obtain equivalent results from cut-off and dimensional regularizations.

However, there are reasons to argue against the use of energy-dependent bare couplings. In field theory they correspond to counterterms that violate time reversal invariance, and also destroy the usual Hermitian structure of the quantum-mechanical potential generating the $S$ matrix. In spite of these, we shall use energy-dependent bare couplings in this study, as that seems to be a means for obtaining equivalent results from cut-off and dimensional renormalization as the ultraviolet divergence is energy dependent in this case. The renormalized physical result should be independent of the regularization schemes employed. For $L>0$, because of the additional freedom provided by the energydependent couplings, we can obtain equivalent results from the two different regularization schemes.

Employing bare coupling (16), the regularized $\tau$ function of Eq. (10) can now be rewritten as

$$
\tau_{R L}(k, \Lambda)=\left[\lambda_{L}^{-1}(k, \Lambda)-I_{R L}(k, \Lambda)\right]^{-1},
$$

where for a finite $\Lambda, I_{R L}(k, \Lambda)$ is a convergent integral. As $\Lambda \rightarrow \infty, \lambda_{L}^{-1}(k, \Lambda)$ of Eq. (16) has the appropriate divergent behavior, which cancels the divergent part Eq. (15) of $I_{R L}(k, \Lambda)$. As $\Lambda \rightarrow \infty$, one obtains the following renormalized $\tau$ function from Eqs. (15), (16), and (17):

$$
\tau_{\mathcal{R} L}(k)=\lim _{\Lambda \rightarrow \infty}\left[\lambda_{L}^{-1}(k, \Lambda)-I_{R L}(k, \Lambda)\right]^{-1}=\frac{1}{\Lambda_{0 L}\left(k^{2}\right)} .
$$

In three dimensions, the low-energy scattering is usually parametrized by a few parameters as in the effective-range expansion. Hence it is natural to take

$$
\Lambda_{0 L}\left(k^{2}\right)=-1 / a_{L}^{2 L+1}+b_{L}^{1-2 L} k^{2}+\cdots,
$$

where $a_{L}$ is the scattering length and $b_{L}$ is a range parameter. These parameters are usually called physical scales as they measure the physical observables, such as a cross section.

Next, we consider $d=2$. Here the regularized integral $I_{L}(k)$, in the large $\Lambda$ limit $(\Lambda \gg k)$, becomes [6]

$$
\begin{aligned}
I_{R L}(k, \Lambda) & \equiv \mathcal{P} \int q d q q^{2 L} G_{R}\left(q, \Lambda ; k^{2}\right) \\
& =-\left[-\ln \frac{k}{\Lambda}\right], \quad L=0 \\
& =-\left[\frac{\Lambda^{2}}{2}-k^{2} \ln \frac{k}{\Lambda}\right], \quad L=1 \\
& =-\left[\frac{\Lambda^{4}}{4}+\frac{k^{2} \Lambda^{2}}{2}-k^{4} \ln \frac{k}{\Lambda}\right], \quad L=2,
\end{aligned}
$$

so that for a general $L$ we have

$$
I_{R L}(k, \Lambda)=-\sum_{j=1}^{L} \frac{k^{2(L-j)} \Lambda^{2 j}}{2 j}+k^{2 L} \ln \frac{k}{\Lambda} .
$$

In order to obtain a finite renormalized $\tau$ function, the coupling $\lambda_{L}$ should be understood to be the so-called bare coupling defined, for example, by

$$
\lambda_{L}^{-1}(k, \Lambda)=-\sum_{j=1}^{L} \frac{k^{2(L-j)} \Lambda^{2 j}}{2 j}+k^{2 L} \ln \frac{\Lambda_{0 L}\left(k^{2}\right)}{\Lambda},
$$

where the function $\Lambda_{0 L}\left(k^{2}\right)$ can again be used to introduce the physical scale(s) of the system and characterizes the strength of the interaction as in the case with $d=3$. If we use Eqs. (24) and (25) in Eq. (17) the following renormalized $\tau$ function is obtained in the limit $\Lambda \rightarrow \infty$ :

$$
\tau_{\mathcal{R} L}(k)=-\frac{1}{k^{2 L} \ln \left[k / \Lambda_{0 L}\left(k^{2}\right)\right]} .
$$

The $\ln (k)$ dependence in Eq. (26) is the proper low-energy momentum dependence in two dimensions [16]. Expressions (18) and (26) are the renormalized $\tau$ functions obtained with cut-off regularization for $d=3$ and 2, respectively. Now it is realized that the use of energy-dependent bare coupling (16) is essential for removing the ultraviolet divergences in the $\Lambda \rightarrow \infty$ limit by cut-off regularization.

The above-mentioned problem can also be tackled with the help of dimensional regularization [15]. In this procedure integral (11) is evaluated to yield [17]

$$
\begin{aligned}
I_{R L}(k, d) \equiv & \mathcal{P} \int q^{d-1} d q q^{2 L}\left(k^{2}-q^{2}\right)^{-1} \\
= & -\frac{1}{2} \Gamma\left(\frac{2 L+d}{2}\right) \Gamma\left(\frac{2-2 L-d}{2}\right) \\
& \times \operatorname{Re}\left[\left(-k^{2}\right)^{(2 L+d-2) / 2}\right],
\end{aligned}
$$

where Re denotes the real part. Integral (27) is divergent for $d=2$ and 3 and Eq. (28) is the finite result valid for $0<(d$ $+2 L)<2$. In dimensional regularization, Eq. (28) is interpreted to be an extrapolation of the convergent result for small $(d+2 L)(<2)$ to the actual values of $(d+2 L)$ $(\geqslant 2)$ for which the result is divergent. In odd dimensions the dimensionally regularized integral (28) is zero because it 
contains the real part of an imaginary quantity. However, for dimensional regularization in even dimensions, Eq. (28) is used to extract the divergent part of $I_{R L}(k)$ as in Eq. (15) and then renormalization can be performed.

For $d=3$, the dimensionally regularized result (28) is already finite:

$$
I_{R L}(k)=0
$$

and in this case one does not need to introduce a new energydependent bare coupling and from Eqs. (9) and (10) one immediately obtains

$$
\tau_{R L}(k)=\lambda_{L}, \quad \text { and } \quad K_{R L}\left(k^{2}\right)=\lambda_{L} k^{2 L} .
$$

However, equivalence between the cut-off renormalized result (18) and the dimensionally regularized result (30) is obtained if the following energy-dependent bare coupling is used instead:

$$
\lambda_{L}(k)=1 / \Lambda_{0 L}\left(k^{2}\right) .
$$

For $d=2$, the dimensionally regularized result still contains infinities and a subtraction of these infinities is necessary before obtaining a finite renormalized result. In this case, as $d \rightarrow 2$, Eq. (28) can be rewritten as [18]

$$
\begin{aligned}
\lim _{\epsilon \rightarrow 0} I_{R L}(k, \epsilon) & =-k^{2 L}\left[\frac{\Gamma(\epsilon)}{2}\right] \operatorname{Re}\left[\left(-k^{2}\right)^{-\epsilon}\right] \\
& =-k^{2 L}\left[\frac{1}{2 \epsilon}-\ln k-\frac{\gamma}{2}\right],
\end{aligned}
$$

where $\gamma=0.577 \ldots$ is the Euler number and $\epsilon=(1-d / 2)$. In writing Eq. (32), use has been made of the well-known limits

$$
\begin{gathered}
\lim _{\epsilon \rightarrow 0} \Gamma(\epsilon) \rightarrow \frac{1}{\epsilon}-\gamma+\mathcal{O}(\epsilon)+\cdots, \\
\lim _{\epsilon \rightarrow 0} \operatorname{Re}\left[\left(-k^{2}\right)^{-\epsilon}\right] \rightarrow 1-2 \epsilon \ln k+\mathcal{O}\left(\epsilon^{2}\right)+\cdots,
\end{gathered}
$$

and $\Gamma(1+x)=x \Gamma(x)$. In the limit $d \rightarrow 2, \epsilon \rightarrow 0$ and Eq. (32) is divergent. If we compare Eq. (32) with Eq. (24) we find that the $1 / \epsilon$ pole in the dimensionally regularized integral corresponds to the different divergences including a logarithmic divergence in the cut-off regularized integral. Equation (32) contains the logarithm of $k-$ a dimensional variable. The scale of the logarithm is hidden in the $1 / \epsilon$ term and appears when the divergence is canceled with an appropriate choice of bare coupling [18]. For performing renormalization with the dimensionally regularized result (32), one should choose the bare coupling as

$$
\lambda_{L}^{-1}(k, \epsilon)=-\frac{k^{2 L}}{2}\left[\frac{1}{\epsilon}-\gamma\right]+k^{2 L} \ln \Lambda_{0 L}\left(k^{2}\right) .
$$

In the limit $\epsilon \rightarrow 0$, using Eqs. (32) and (35) in

$$
\tau_{R L}(k)=\lim _{\epsilon \rightarrow 0}\left[\lambda_{L}^{-1}(k, \epsilon)-I_{2 L}(k, \epsilon)\right]^{-1}
$$

one obtains the finite renormalized result (26), obtained by cut-off regularization. Hence both regularization schemes yield equivalent renormalized results.

\section{B. The rank-two separable potential}

Next we consider the renormalization of rank-two separable potential (2) for $d=3$. In this case the on-shell $K$ matrix is given by [19]

$$
K\left(k^{2}\right)=\frac{u^{2}(k)\left(1 / \lambda_{2}-A_{v v}\right)+v^{2}(k)\left(1 / \lambda_{0}-A_{u u}\right)+2 u(k) v(k) A_{u v}}{\left(1 / \lambda_{2}-A_{v v}\right)\left(1 / \lambda_{0}-A_{u u}\right)-A_{u v}^{2}},
$$

where

$$
\begin{gathered}
A_{u u} \equiv \mathcal{P} \int q^{2} d q u^{2}(q) G\left(q ; k^{2}\right), \\
A_{u v} \equiv \mathcal{P} \int q^{2} d q u(q) v(q) G\left(q ; k^{2}\right), \\
A_{v v} \equiv \mathcal{P} \int q^{2} d q v^{2}(q) G\left(q ; k^{2}\right) .
\end{gathered}
$$

In order to work out the divergent terms explicitly we consider a specific case: $u(p)=1, v(p)=p^{2}$. This specialization does not correspond to any real loss of generality. Other choices of $u(p)$ and $v(p)$ can be worked out similarly. With this choice the $K$ matrix becomes

$$
K\left(k^{2}\right)=\frac{\left[1 / \lambda_{2}-I_{2}(k)\right]+k^{4}\left[1 / \lambda_{0}-I_{0}(k)\right]+2 k^{2} I_{1}(k)}{\left[1 / \lambda_{2}-I_{2}(k)\right]\left[1 / \lambda_{0}-I_{0}(k)\right]-I_{1}^{2}(k)} .
$$

In this case, divergent integrals $A_{u u}, A_{u v}$, and $A_{v v}$ are $I_{L}(k)$ of Eq. (11) with $L=0,1$, and 2, respectively. These have been treated by dimensional and cut-off regularizations in Sec. II A. Hence one can use the same regularization procedure as employed there. However, in general, $\lambda_{0}$ and $\lambda_{2}$ of Eq. (41) are to be interpreted as cut-off $(\Lambda)$ dependent bare coupling.

In this case first we perform dimensional regularization. From Eq. (29), we find that the dimensional regularization of integrals $I_{L}(k)$ for $d=3$ are all zero. Then, if we use the energy-dependent bare couplings (31), from Eq. (41) we obtain the following finite regularized on-shell $K$ matrix: 


$$
K_{R}\left(k^{2}\right)=\Lambda_{00}^{-1}\left(k^{2}\right)+\Lambda_{02}^{-1}\left(k^{2}\right) k^{4},
$$

where $\Lambda_{0 L}^{-1}\left(k^{2}\right)$ 's are polynomials in $k^{2}$ as in Eq. (19). The renormalized $K$ matrix (42) will have the form of a polynomial in $k^{2}$ at low energies.

Next we employ cut-off regularization in Eq. (41). If we use Eq. (15), regularized $K$ matrix (41) becomes

$$
\begin{aligned}
K_{R}\left(k^{2}\right) & =\frac{A+B k^{2}+C k^{4}}{D+E k^{2}+F k^{4}} \\
& =\frac{A}{D}+\left(\frac{B}{D}-\frac{A E}{D^{2}}\right) k^{2}+\cdots,
\end{aligned}
$$

where

$$
\begin{gathered}
A=\frac{1}{\lambda_{2}}+\frac{\Lambda^{5}}{5}, \quad B=-\frac{\Lambda^{3}}{3}, \quad C=\frac{1}{\lambda_{0}}, \\
D=\frac{1}{\lambda_{0} \lambda_{2}}+\frac{\Lambda^{5}}{5 \lambda_{0}}+\frac{\Lambda}{\lambda_{2}}+\frac{4 \Lambda^{6}}{45}, \\
E=\frac{\Lambda^{3}}{3 \lambda_{0}}-\frac{\Lambda^{4}}{3}, \quad \text { and } F=\frac{\Lambda}{\lambda_{0}} .
\end{gathered}
$$

Equation (43) represents the exact solution and Eq. (44) is its low-energy expansion. One performs renormalization subject to conditions $k \rightarrow 0$ and $\Lambda \rightarrow \infty$. As there are two couplings $\lambda_{0}$ and $\lambda_{2}$, two renormalization conditions can be used in this case. These two conditions can be used to determine the constant term and the coefficient of the $k^{2}$ term in Eq. (44). Thus one introduces two renormalized parameters via

$$
\lambda_{0}^{R}=\lim _{\Lambda \rightarrow \infty} \frac{A}{D}, \quad \lambda_{2}^{R}=\lim _{\Lambda \rightarrow \infty}\left(\frac{B}{D}-\frac{A E}{D^{2}}\right)
$$

so that the renormalized $K$ matrix becomes

$$
K_{R}\left(k^{2}\right)=\lambda_{0}^{R}+\lambda_{2}^{R} k^{2}+\cdots,
$$

which is the result of cut-off renormalization at low energies up to terms linear in $k^{2}$. Equations (47) define the bare couplings in the limit $\Lambda \rightarrow \infty$, although it is not possible to write closed-form expressions for them in this case.

In this case it is possible to guarantee the equivalence of the $K$ matrices (42) and (43). For this purpose, it is necessary to take advantage of the energy-dependence of the bare couplings to ensure

$$
\Lambda_{00}^{-1}\left(k^{2}\right)+\Lambda_{02}^{-1}\left(k^{2}\right) k^{4}=\lim _{\Lambda \rightarrow \infty} \frac{A+B k^{2}+C k^{4}}{D+E k^{2}+F k^{4}},
$$

where some care must be taken since bare couplings $\lambda_{0}$ and $\lambda_{2}$ depend on $\Lambda$. One can also enforce equivalence by writing $\lambda_{0}$ and $\lambda_{2}$ as energy-dependent functions of $\Lambda_{00}$ and $\Lambda_{02}$. A suitable choice of bare couplings will give the equivalence of the two results.

\section{A $\delta$-function potential and its derivatives}

Now we consider potential (3) for $d=3$, which is the sum of a $\delta$ function and its second derivatives in configuration space. This potential appears in the field theoretic reduction of low-energy nucleon-nucleon potential $[5,6,11,13]$. After a straightforward calculation, the on-shell $K$ matrix for this potential is given by

$$
\begin{aligned}
& K\left(k^{2}\right) \\
& \quad=\frac{2 k^{2} / \lambda_{2}+\lambda_{1} / \lambda_{2}^{2}+I_{2}(k)-2 k^{2} I_{1}(k)+k^{4} I_{0}(k)}{1 / \lambda_{2}^{2}-2 I_{1}(k) / \lambda_{2}+I_{1}^{2}(k)-\lambda_{1} I_{0}(k) / \lambda_{2}^{2}-I_{0}(k) I_{2}(k)},
\end{aligned}
$$

where $I_{L}(k)$ 's are given by Eq. (11). These integrals have been treated by dimensional and cut-off regularizations in Sec. II A. Hence one can use the same regularization procedure as employed there. Again, $\lambda_{1}$ and $\lambda_{2}$ of Eq. (50) are to be interpreted as cut-off $(\Lambda)$ dependent bare coupling.

Here, first we perform dimensional regularization. From Eq. (29), we find that the dimensional regularization of integrals $I_{L}(k)$ for $d=3$ are all zero. Then, if we use the energydependent bare couplings (31), from Eq. (41) one obtains the following finite regularized on-shell $K$ matrix:

$$
K_{R}\left(k^{2}\right)=\Lambda_{1}^{-1}\left(k^{2}\right)+\Lambda_{2}^{-1}\left(k^{2}\right) k^{2},
$$

where $\Lambda_{1}^{-1}\left(k^{2}\right)$ and $\Lambda_{2}^{-1}\left(k^{2}\right)$ are polynomials in $k^{2}$ as in Eq. (19). Hence the renormalized $K$ matrix (51) has the form of a polynomial in $k^{2}$ at low energies.

Next we employ cut-off regularization in Eq. (50). Then the quantities $I_{0}(k), I_{2}(k)$, and $I_{4}(k)$ are, respectively, given by Eq. (15) with $L=0,1$, and 2, and we obtain from Eq. (50)

$$
\begin{aligned}
K_{R}\left(k^{2}\right) & =\frac{A+B k^{2}}{D+E k^{2}} \\
& =\frac{A}{D}+\left(\frac{B}{D}-\frac{A E}{D^{2}}\right) k^{2}+\cdots,
\end{aligned}
$$

where

$$
\begin{gathered}
A=\frac{\lambda_{1}}{\lambda_{2}^{2}}-\frac{\Lambda^{5}}{5}, \quad B=\frac{\Lambda^{3}}{3}+\frac{2}{\lambda_{2}}, \\
D=\frac{1}{\lambda_{2}^{2}}+\frac{2 \Lambda^{3}}{3 \lambda_{2}}+\frac{\lambda_{1} \Lambda}{\lambda_{2}^{2}}-\frac{4 \Lambda^{6}}{45}, \quad \text { and } \quad E=\frac{2 \Lambda}{\lambda_{2}}+\frac{\Lambda^{4}}{3} .
\end{gathered}
$$

Equation (52) represents the exact solution and Eq. (53) is its low-energy expansion. Now as in Sec. II B one could introduce two renormalized parameters via Eq. (47) and obtain a cut-off renormalized $K$ matrix at low energies as in Eq. (48). The cut-off renormalized $K$ matrix can be consistent with the dimensionally renormalized one (51), if one exploits the flexibility introduced by the energy-dependent bare couplings to ensure 


$$
\Lambda_{1}^{-1}\left(k^{2}\right)+\Lambda_{2}^{-1}\left(k^{2}\right) k^{2}=\lim _{\Lambda \rightarrow \infty} \frac{A+B k^{2}}{D+E k^{2}},
$$

where again some care must be taken since bare couplings $\lambda_{0}$ and $\lambda_{2}$ depend on $\Lambda$.

\section{The tensor potential}

Finally, we consider the renormalization of the tensor potential (4) in three dimensions. The $K$ matrix elements in this case satisfy the following set of coupled equations:

$$
\begin{aligned}
K_{L L^{\prime}}\left(p, k, k^{2}\right)= & V_{L L^{\prime}}(p, k)+\mathcal{P} \sum_{L^{\prime \prime}=0,2} \int q^{2} d q V_{L L^{\prime \prime}}(p, q) \\
& \times G\left(q ; k^{2}\right) K_{L^{\prime \prime} L^{\prime}}\left(q, k, k^{2}\right) .
\end{aligned}
$$

From Eqs. (4) and (57) it is realized that the $K$ matrix elements have the following form:

$$
\begin{aligned}
\left|K_{L L^{\prime}}\left(p, q, k^{2}\right)\right| & \equiv\left(\begin{array}{cc}
K_{00}\left(p, q, k^{2}\right) & K_{02}\left(p, q, k^{2}\right) \\
K_{20}\left(p, q, k^{2}\right) & K_{22}\left(p, q, k^{2}\right)
\end{array}\right) \\
& =\left(\begin{array}{cc}
\tau_{0} & \tau_{1} q^{2} \\
\tau_{1} p^{2} & \tau_{2} p^{2} q^{2}
\end{array}\right),
\end{aligned}
$$

where the energy-dependent functions $\tau$ 's are defined by

$$
\begin{aligned}
\left(\begin{array}{cc}
\tau_{0} & \tau_{1} \\
\tau_{1} & \tau_{2}
\end{array}\right)= & \left(\begin{array}{cc}
\lambda_{0} & \lambda_{1} \\
\lambda_{1} & \lambda_{2}
\end{array}\right) \\
& +\left(\begin{array}{cc}
\lambda_{0} & \lambda_{1} \\
\lambda_{1} & \lambda_{2}
\end{array}\right)\left(\begin{array}{cc}
I_{0}(k) & 0 \\
0 & I_{2}(k)
\end{array}\right)\left(\begin{array}{cc}
\tau_{0} & \tau_{1} \\
\tau_{1} & \tau_{2}
\end{array}\right),
\end{aligned}
$$

with $I_{0}(k)$ and $I_{2}(k)$ given by Eq. (11).

Equation (59) can be rewritten as

$$
\left(\begin{array}{cc}
1-\lambda_{0} I_{0}(k) & -\lambda_{1} I_{2}(k) \\
-\lambda_{1} I_{0}(k) & 1-\lambda_{2} I_{2}(k)
\end{array}\right)\left(\begin{array}{cc}
\tau_{0} & \tau_{1} \\
\tau_{1} & \tau_{2}
\end{array}\right)=\left(\begin{array}{cc}
\lambda_{0} & \lambda_{1} \\
\lambda_{1} & \lambda_{2}
\end{array}\right) .
$$

The following solution of Eq. (60) can be obtained after straightforward algebra:

$$
\left(\begin{array}{cc}
\tau_{0} & \tau_{1} \\
\tau_{1} & \tau_{2}
\end{array}\right)=\frac{1}{\mathcal{D}}\left(\begin{array}{cc}
\lambda_{0}+\left(\lambda_{1}^{2}-\lambda_{0} \lambda_{2}\right) I_{2}(k) & \lambda_{1} \\
\lambda_{1} & \lambda_{2}+\left(\lambda_{1}^{2}-\lambda_{0} \lambda_{2}\right) I_{0}(k)
\end{array}\right),
$$

where

$$
\mathcal{D} \equiv\left[1-\lambda_{0} I_{0}(k)\right]\left[1-\lambda_{2} I_{2}(k)\right]-\lambda_{1}^{2} I_{0}(k) I_{2}(k)
$$

is the determinant of the first matrix on the left-hand side of Eq. (60). If we use a dimensionally regularized result (29) for integrals $I_{0}(k)$ and $I_{2}(k)$ in Eqs. (61) and (62), we obtain the following dimensionally regularized $\tau$ function:

$$
\left(\begin{array}{cc}
\tau_{R 0} & \tau_{R 1} \\
\tau_{R 1} & \tau_{R 2}
\end{array}\right)=\left(\begin{array}{cc}
\Lambda_{0}^{-1}\left(k^{2}\right) & \Lambda_{1}^{-1}\left(k^{2}\right) \\
\Lambda_{1}^{-1}\left(k^{2}\right) & \Lambda_{2}^{-1}\left(k^{2}\right)
\end{array}\right),
$$

where we have employed the usual energy-dependent bare couplings.

Next we employ cut-off regularization to Eq. (61). If we use the cut-off regularized result (15) for integrals $I_{L}(k)$, each of $\tau_{0}, \tau_{1}$, and $\tau_{2}$ will have a form similar to the righthand side of Eq. (43) and one can make an expansion in $k^{2}$ as in Eq. (44). Using explicit forms of cut-off regularized integrals $I_{L}(k)$, the regularized $\tau_{0}, \tau_{1}$, and $\tau_{2}$ can be written as

$$
\begin{gathered}
\tau_{R 0}=J\left[\lambda_{0}-\left(\lambda_{1}^{2}-\lambda_{0} \lambda_{2}\right) \Lambda^{5} / 5\right]+\mathcal{O}\left(k^{2}\right)+\cdots, \\
\tau_{R 1}=J \lambda_{1}+\mathcal{O}\left(k^{2}\right)+\cdots, \\
\tau_{R 2}=J\left[\lambda_{2}-\left(\lambda_{1}^{2}-\lambda_{0} \lambda_{2}\right) \Lambda\right]+\mathcal{O}\left(k^{2}\right)+\cdots,
\end{gathered}
$$

where

$$
J=\left[1+\lambda_{0} \Lambda+\lambda_{2} \Lambda^{5} / 5-\left(\lambda_{1}^{2}-\lambda_{0} \lambda_{2}\right) \Lambda^{6} / 5\right]^{-1} .
$$

In this case there are three couplings in potential (4): $\lambda_{0}, \lambda_{1}$, and $\lambda_{2}$. These couplings are to be interpreted as the bare couplings for regularization and renormalization. Now if one introduces three renormalized couplings $\lambda_{R 0}, \lambda_{R 1}$, and $\lambda_{R 2}$ through

$$
\begin{gathered}
\lim _{\Lambda \rightarrow \infty} J\left[\lambda_{0}-\left(\lambda_{1}^{2}-\lambda_{0} \lambda_{2}\right) \Lambda^{5} / 5\right]=\lambda_{R 0}, \\
\lim _{\Lambda \rightarrow \infty} J \lambda_{1}=\lambda_{R 1}, \\
\lim _{\Lambda \rightarrow \infty} J\left[\lambda_{2}-\left(\lambda_{1}^{2}-\lambda_{0} \lambda_{2}\right) \Lambda\right]=\lambda_{R 2},
\end{gathered}
$$

one obtains the following energy-independent solution valid in the extreme low-energy limit:

$$
\left(\begin{array}{cc}
\tau_{R 0} & \tau_{R 1} \\
\tau_{R 1} & \tau_{R 2}
\end{array}\right)=\left(\begin{array}{cc}
\lambda_{R 0} & \lambda_{R 1} \\
\lambda_{R 1} & \lambda_{R 2}
\end{array}\right)
$$

Again it is possible to establish equivalence between results (63) and (71). 


\section{NUMERICAL STUDY}

The renormalization of the sum of a finite-range and a divergent potential is of considerable interest in the context of effective-field-theoretic nucleon-nucleon interaction $[7,9,11,12]$. Such a sum of divergent and finite-range potentials appears in a field-theoretic description of low-energy nucleon-nucleon interaction as in Eq. (5) [11,13]. It is not even clear whether such potentials could be renormalized successfully. We address this point in the following, where the finite-range potential is taken to be a separable one. A general discussion with a complicated finite-range potential will only add to numerical complication. We perform renormalization when a finite-range attractive separable potential, $V_{f}(p, q)=\lambda_{1} u(p) u(q)$ with $u(p)=\left[\alpha^{2} /\left(\alpha^{2}+p^{2}\right)\right]^{2}, \quad$ is added to a divergent potential, $V_{d}(p, q)$. Among divergent potentials we specifically consider $V_{d}(p, q)=\lambda_{2}$ or $V_{d}(p, q)=\lambda_{2} p^{2} q^{2}$, which appears in Eq. (5).

The $K$ matrix for finite-range separable potential $V_{f}(p, q)$ is

$$
K\left(k^{2}\right)=\frac{u^{2}(k)}{1 / \lambda_{1}-A_{u u}},
$$

with $A_{u u}$ given by Eq. (38). Potential $V_{f}(p, q)$ is taken to model an $S$-wave spin-triplet nucleon-nucleon interaction.

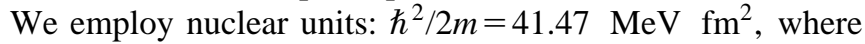
$2 m$ is the nucleon mass and take $\alpha^{2}=1 \mathrm{fm}^{-2}$. We assume that $V_{f}(p, q)$ supports a bound deuteron and produces the experimental scattering length $a \equiv \pi K\left(k^{2}\right) / 2=5.42 \mathrm{fm}$. This fixes the value of coupling to be $\lambda_{1} \equiv \lambda_{0}=-4.9737 \mathrm{fm}$.

We consider the sum of divergent potential $V_{d}(p, q)$, and the separable potential, $V_{f}(p, q)$, given by Eq. (3) with $v(p)=1$ or $v(p)=p^{2}$ and $u(p)$ defined above. The formal solution for the $K$ matrix with this potential is given by Eq. (37), where the only divergent integral is $A_{v v}$. In order to obtain a regularized $K$ matrix, we introduce a bare coupling in Eq. (37) in place of $\lambda_{2}$ such that $1 / \lambda_{2}^{R} \equiv\left(1 / \lambda_{2}-A_{v v}\right)$ is a finite quantity, where $\lambda_{2}^{R}$ is the renormalized coupling which is taken to be energy independent. Then one obtains the following regularized $K$ matrix:

$$
\begin{aligned}
& K_{R}\left(k^{2}\right) \\
& =\frac{u^{2}(k)\left(1 / \lambda_{2}^{R}\right)+v^{2}(k)\left(1 / \lambda_{1}-A_{u u}\right)+2 u(k) v(k) A_{u v}}{\left(1 / \lambda_{2}^{R}\right)\left(1 / \lambda_{1}-A_{u u}\right)-A_{u v}^{2}},
\end{aligned}
$$

which is appropriate for both cut-off and dimensional regularizations. Of course, in dimensional regularization, $A_{v v}$ $=0$ and $\lambda_{2}^{R}=\lambda_{2}$, if energy-independent bare coupling is used. If we demand that regularized $K$ matrix (73) should yield identical phase-shift or scattering length as that produced by potential $V_{f}(p, q)$, we find that $\lambda_{2}^{R}=\lambda_{2}=0$, which sets the divergent potential equal to zero. In order that Eq. (73) still models the $S$-wave nucleon-nucleon triplet $K$ matrix in the presence of a nonzero divergent potential, either the strength $\lambda_{1}$ or the parameter $\alpha$ of the separable potential should be changed once the divergent potential is included. We keep the parameter $\alpha=1 \mathrm{fm}^{-2}$ unchanged and consider two possibilities for the coupling $\lambda_{1}$ of $V_{f}(p, q)$ in the pres-

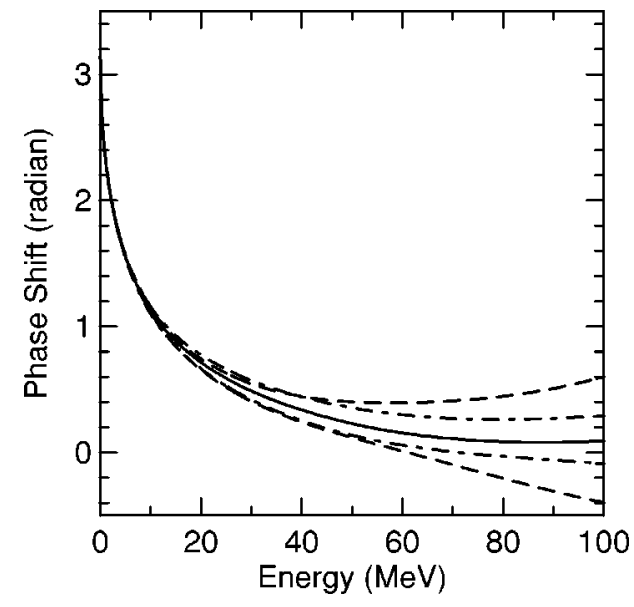

FIG. 1. Phase shifts at different center-of-mass energies for (a) separable potential $V_{f}(p, q)$ (solid line) with coupling $\lambda_{0}$ $=-4.9737 \mathrm{fm}$, (b) potential $V_{f}(p, q)+\lambda_{2}$ (dashed-dotted line), and (c) potential $V_{f}(p, q)+\lambda_{2} p^{2} q^{2}$ (dashed line). The phase shifts (b) and (c) refer to renormalized $K$ matrices. Of the two curves in sets (b) and (c) the upper (lower) one refers to separable potential coupling $\lambda_{1}=0.9 \lambda_{0}\left(1.1 \lambda_{0}\right)$.

ence of the divergent potentials: $\lambda_{1}=1.1 \lambda_{0}=-5.4711 \mathrm{fm}$ and $\lambda_{1}=0.9 \lambda_{0}=-4.4763 \mathrm{fm}$. In order that the renormalized $K$ matrix (73) leads to the scattering length $5.42 \mathrm{fm}$, one should have $\lambda_{2}^{R}=0.0729 \mathrm{fm}(0.0296 \mathrm{fm})$ for $\lambda_{1}=1.1 \lambda_{0}$, and $\lambda_{2}^{R}=-0.0934 \mathrm{fm}(-0.0362 \mathrm{fm})$ for $\lambda_{1}=0.9 \lambda_{0}$ with the form-factor of divergent potential $v(p)=1\left(p^{2}\right)$. In the case where the strength of the attractive separable interaction $V_{f}(p, q)$ is increased (decreased), the renormalized strength of the divergent potential should be repulsive (attractive) in order to reproduce the experimental scattering length 5.42 $\mathrm{fm}$. This is reflected by the sign of the renormalized strength $\lambda_{2}^{R}$ of the divergent potential in both cases.

The phase shifts of different schemes are shown in Fig. 1, where we plot phase shifts for potential $V_{f}(p, q)$ (solid line) and the two sets of renormalized phase shifts in the presence of the divergent potentials $\lambda_{2}$ (dashed-dotted line) and $\lambda_{2} p^{2} q^{2}$ (dashed line) for $\lambda_{1}=1.1 \lambda_{0}$ and $\lambda_{1}=0.9 \lambda_{0}$. The two lines below (above) the solid line correspond to $\lambda_{1}=1.1 \lambda_{0}$ $\left(0.9 \lambda_{0}\right)$.

In the presence of ultraviolet divergences, any renormalization scheme should lead to physically plausible result at low energies, with energies much lower than the cutoff. Hence the two versions of renormalized $K$ matrices (73) should be similar to $K$ matrix (72) at low energies. From Fig. 1 we find that this is indeed the case. An examination of $K$ matrix (72) reveals that it tends to zero as $k^{2} \rightarrow \infty$, which is physically expected from scattering equation (6). However, $K$ matrix (73) tends to $\lambda_{2}^{R}\left(\lambda_{2}^{R} k^{4}\right)$ as $k^{2} \rightarrow \infty$ for $v(p)$ $=1\left(p^{2}\right)$. Hence at high energies the renormalized $K$ matrix has physically unacceptable behavior. However, Eq. (73) yields very reasonable result at low energies, which define the domain of validity of renormalization. In the presence of the divergent potential a new parametrization of the interaction is needed to fit the experimental observables. In order to fit the phase shifts to higher energies, specially in the presence of stronger ultraviolet divergence, the quantity $1 / \lambda_{2}^{R}$ $\equiv\left(1 / \lambda_{2}-A_{v v}\right)$ has to be taken to be $k^{2}$ dependent with several parameters. 


\section{SUMMARY}

We have renormalized the $K$ matrices obtained with potentials (1), (2), (3), and (4) by cut-off and dimensional regularizations. The solution of the dynamical problem in these cases involves ultraviolet divergences. For these potentials in three dimensions, all dimensionally regularized divergent integrals over Green's functions are identically zero. Both cutoff and dimensional regularization schemes lead to equivalent renormalized results for potentials (1), (2), and (3), only if general energy-dependent bare couplings are employed. For tensor potential (4), both regularizations in three dimensions also lead to equivalent $K$ matrices. We also performed renormalization when the potential is the sum of a separable and an analytic divergent potential. For the divergent parts we took one of the following two potentials: $\lambda$ and $\lambda p^{2} q^{2}$.
The renormalized result is taken to simulate the $S$-wave spintriplet nucleon-nucleon potential. We find that in both cases the low-energy renormalized phase shifts are physically plausible. The present analytical and numerical studies demonstrate that regularization and renormalization are efficient tools for treating divergent potentials in nonrelativistic quantum mechanics.

\section{ACKNOWLEDGMENTS}

We thank Dr. Rabin Banerjee and Dr. Marcelo de M. Leite for informative discussions and the Conselho Nacional de Desenvolvimento Científico e Tecnológico, Fundação de Amparo à Pesquisa do Estado de São Paulo, Financiadora de Estudos e Projetos of Brazil, and the John Simon Guggenheim Memorial Foundation for partial financial support.
[1] S. K. Adhikari and A. Ghosh, J. Phys. A 30, 6553 (1997).

[2] S. K. Adhikari and T. Frederico, Phys. Rev. Lett. 74, 4572 (1995); S. K. Adhikari, T. Frederico, and I. D. Goldman, ibid. 74, 487 (1995); C. F. de Araujo, Jr., L. Tomio, S. K. Adhikari, and T. Frederico, J. Phys. A 30, 4687 (1997).

[3] R. Jackiw, in M. A. B. Beg Memorial Volume, edited by A. Ali and P. Hoodbhoy (World Scientific, Singapore, 1991), pp. 2542; C. Manuel and R. Tarrach, Phys. Lett. B 328, 113 (1994); L. R. Mead and J. Godines, Am. J. Phys. 59, 935 (1991); M. A. B. Beg and R. C. Furlong, Phys. Rev. D 31, 1370 (1985); T. J. Fields, K. S. Gupta, and J. P. Vary, Mod. Phys. Lett. A 11, 2233 (1996); G. Amelino-Camelia, Phys. Lett. B 326, 282 (1994); Phys. Rev. D 51, 2000 (1995); T. D. Cohen, Phys. Rev. C 55, 67 (1997); H. El Hattab and J. Polonyi, preprint, hep-th/9711061.

[4] S. K. Adhikari, T. Frederico, and R. M. Marinho, J. Phys. A 29, 7157 (1996).

[5] D. R. Phillips, S. R. Beane, and T. D. Cohen, Nucl. Phys. A632, 445 (1998).

[6] D. R. Phillips, S. R. Beane, and T. D. Cohen, Ann. Phys. (N.Y.) 263, 255 (1998).

[7] K. A. Scaldeferri, D. R. Phillips, C. W. Kao, and T. D. Cohen, Phys. Rev. C 56, 679 (1997).

[8] M. Luke and A. V. Manohar, Phys. Rev. D 55, 4129 (1997); P. Bedaque and U. van Kolck, Phys. Lett. B 428, 221 (1998); D. B. Kaplan, Nucl. Phys. B494, 471 (1997); D. B. Kaplan, M. J. Savage, and M. B. Wise, preprint, nucl-th/9801034.

[9] G. P. Lepage, preprint, nucl-th/9706029.
[10] K. G. Richardson, M. C. Birse, and J. A. McGovern, preprint, hep-ph/9708435; M. C. Birse, J. A. McGovern, and K. G. Richardson, preprint, hep-ph/9807302; hep-ph/9808398.

[11] D. B. Kaplan, M. J. Savage, and M. B. Wise, Nucl. Phys. B478, 629 (1996).

[12] C. Ordonéz, L. Ray, and U. van Kolck, Phys. Rev. C 53, 2086 (1996).

[13] S. Weinberg, Nucl. Phys. B363, 3 (1991); Phys. Lett. B 251, 288 (1990); Physica A 96, 327 (1979); E. Witten, Nucl. Phys. B122, 109 (1977).

[14] K. G. Wilson and J. Kogut, Phys. Rep., Phys. Lett. 12C, 78 (1974); L. H. Ryder, Quantum Field Theory (Cambridge University Press, Cambridge, 1985); P. Ramond, Field Theory: A Modern Primer (Benjamin/Cummings, Reading, MA, 1981); S. Weinberg, The Quantum Theory of Fields (Cambridge University Press, New York, 1995).

[15] C. G. Bollini and J. J. Giambiagi, Phys. Lett. 40B, 566 (1972); Nuovo Cimento B 12, 20 (1972); G. 't Hooft and M. Veltman, Nucl. Phys. B44, 189 (1972).

[16] S. K. Adhikari, Am. J. Phys. 54, 362 (1986); S. K. Adhikari, W. G. Gibson, and T. K. Lim, J. Chem. Phys. 85, 5580 (1986).

[17] I. S. Gradshteyn and I. M. Ryzhik, Table of Integrals, Series, and Products (Academic, New York, 1965), p. 292, equation 3.241.4.

[18] M. E. Peskin and D. V. Schroeder, An Introduction to Quantum Field Theory (Addison-Wesley, Reading, 1995), p. 250.

[19] R. D. Amado, in Elementary Particle Physics and Scattering Theory, edited by M. Chretien and S. S. Schweber (Gordon and Breach, New York, 1970), Vol. 2, p. 40. 